\title{
Periprosthetic UHMWPE Wear Debris Induces Inflammation, Vascularization, and Innervation After Total Disc Replacement in the Lumbar Spine
}

\author{
Sai Y. Veruva PhD, Todd H. Lanman MD, Jorge E. Isaza MD, \\ Theresa A. Freeman PhD, Steven M. Kurtz PhD, \\ Marla J. Steinbeck MT(ASCP), PhD
}

Published online: 3 August 2016

(C) The Association of Bone and Joint Surgeons (B) 2016

\begin{abstract}
Background The pathophysiology and mechanisms driving the generation of unintended pain after total disc replacement (TDR) remain unexplored. Ultrahigh-molecular-weight polyethylene (UHMWPE) wear debris from TDRs is known to induce inflammation, which may result in pain.
\end{abstract}

Funding provided by the National Institutes of Health (NIAMS) R01 AR56264 (SMK, MJS). One of the authors (SMK) is an officer and shareholder of Exponent, Inc (Philadelphia, PA, USA), and institutional support for SMK is received as a Principal Investigator from Smith \& Nephew (Memphis, TN, USA); Stryker Orthopaedics (Mahwah, NJ, USA); Zimmer Inc (Warsaw, IN, USA); Biomet (Warsaw, IN, USA); DePuy Synthes (West Chester, PA, USA); Medtronic (Minneapolis, MN, USA); Invibio (Lancashire, UK); Stelkast (McMurray, PA, USA); Formae (Paoli, PA, USA); Kyocera Medical (Osaka, Japan); Wright Medical Technology (Memphis, TN, USA); CeramTec (Plochingen, Germany); DJO (Austin, TX, USA); Celanese (Bedminster Township, NJ, USA); Aesculap (Tuttlingen, Germany); SpinalMotion, Inc (Mountain View, CA, USA); Active Implants (Memphis, TN, USA); and Ferring Pharmaceuticals (Parsipanny, NJ, USA) outside of the submitted work. One of the authors (THL) certifies that he has received royalties during the study period of USD 100,001 to USD 1,000,000 from Medtronic (Memphis, TN, USA).

All ICMJE Conflict of Interest Forms for authors and Clinical Orthopaedics and Related Research ${ }^{\circledR}$ editors and board members are on file with the publication and can be viewed on request.

Clinical Orthopaedics and Related Research ${ }^{\circledR}$ neither advocates nor endorses the use of any treatment, drug, or device. Readers are encouraged to always seek additional information, including FDAapproval status, of any drug or device prior to clinical use.

Each author certifies that his or her institution approved or waived approval for the human protocol for this investigation and that all investigations were conducted in conformity with ethical principles of research.

This work was performed at the Implant Research Center,

Philadelphia, PA, USA.
Questions/purposes The purpose of this study was to determine whether (1) periprosthetic UHMWPE wear debris induces immune responses that lead to the production of tumor necrosis factor- $\alpha(\mathrm{TNF} \alpha)$ and interleukin (IL)-1B, the vascularization factors, vascular endothelial growth factor (VEGF) and platelet-derived growth factorbb (PDGFbb), and the innervation/pain factors, nerve growth factor (NGF) and substance P; (2) the number of

Electronic supplementary material The online version of this article (doi:10.1007/s11999-016-4996-8) contains supplementary material, which is available to authorized users.

S. Y. Veruva, S. M. Kurtz, M. J. Steinbeck $(\square)$

Implant Research Center, Drexel University, 3401 Market Street,

Suite 345, Philadelphia, PA 19104, USA

e-mail:mjs348@drexel.edu

T. H. Lanman

Department of Neurosurgery, UCLA David Geffen School of

Medicine, Los Angeles, CA, USA

J. E. Isaza

Tulane University, Baton Rouge, LA, USA

T. A. Freeman

Department of Orthopaedic Surgery, Thomas Jefferson

University, Philadelphia, PA, USA

S. M. Kurtz

Exponent, Inc, Philadelphia, PA, USA

M. J. Steinbeck

Department of Orthopaedic Surgery, Drexel University College of Medicine, Philadelphia, PA, USA 
macrophages is associated with the production of the aforementioned factors; (3) the wear debris-induced inflammatory pathogenesis involves an increase in vascularization and associated innervation.

Methods Periprosthetic tissues from our collection of 11 patients with contemporary TDRs were evaluated using polarized light microscopy to quantify UHMWPE wear particles. The major reason for revision (mean implantation time of 3 years [range, 1-6 years]) was pain. For control subjects, biopsy samples from four patients with degenerative disc disease with severe pain and autopsy samples from three normal patients with no history of back pain were also investigated. Immunohistochemistry and histology were used to identify secretory factors, macrophages, and blood vessels. Immunostained serial sections were imaged at $\times 200$ magnification and using MATLAB and NIH ImageJ, a threshold was determined for each factor and used to quantify positive staining normalized to tissue sectional area. The Mann-Whitney U test was used to compare results from different patient groups, whereas the Spearman Rho test was used to determine correlations. Significance was based on $\mathrm{p}<$ 0.05 .

Results The mean percent area of all six inflammatory, vascularization, and innervation factors was higher in TDR tissues when compared with normal disc tissues. Based on nonparametric data analysis, those factors showing the most significant increase included $\mathrm{TNF} \alpha(5.17 \pm 1.76$ versus $0.05 \pm 0.03, \mathrm{p}=0.02)$, VEGF $(3.02 \pm 1.01$ versus $0.02 \pm 0.002, \mathrm{p}=0.02)$, and substance $\mathrm{P}(4.15 \pm 1.01$ versus $0.08 \pm 0.04, p=0.02$ ). The mean percent area for IL-1ß $(2.41 \pm 0.66$ versus $0.13 \pm 0.13, \mathrm{p}=0.01)$, VEGF $(3.02 \pm 1.01$ versus $0.34 \pm 0.29, \mathrm{p}=0.04)$, and substance $\mathrm{P}$ $(4.15 \pm 1.01$ versus $1.05 \pm 0.46, p=0.01)$ was also higher in TDR tissues when compared with disc tissues from patients with painful degenerative disc disease. Five of the factors, TNF $\alpha$, IL-1ß, VEGF, NGF, and substance P, strongly correlated with the number of wear particles, macrophages, and blood vessels. The most notable correlations included TNF $\alpha$ with wear particles $(\mathrm{p}<0.001, \rho=$ $0.63)$, VEGF with macrophages $(\mathrm{p}=0.001, \rho=0.71)$, and NGF with blood vessels $(p<0.001, \rho=0.70)$. Of particular significance, the expression of PDGFbb, NGF, and substance $\mathrm{P}$ was predominantly localized to blood vessels/ nerve fibers.

Conclusions These findings indicate wear debris-induced inflammatory reactions can be linked to enhanced vascularization and associated innervation/pain factor production at periprosthetic sites around TDRs. Elucidating the pathogenesis of inflammatory particle disease will provide information needed to identify potential therapeutic targets and treatment strategies to mitigate pain and potentially avoid revision surgery.
Level of Evidence Level III, therapeutic study.

\section{Introduction}

The pathophysiology of low back pain remains poorly understood [25], and even less is known about the mechanism(s) involved in the generation of unexplained pain after metal-on-UHMWPE total disc replacement (TDR). The normal human lumbar disc consists of an avascular/ aneural nucleus pulposus and a surrounding annulus fibrosus that is poorly vascularized and innervated [21]. Painful disc degeneration is associated with the infiltration of inflammatory cells and responses leading to innervation by sensory nerve fibers, which follow the path of ingrowing blood vessels into disc tissue [4, 9]. This process is mediated by activated fibroblasts, inflammatory cells, and other resident disc cells that release factors that can lead to pain sensitization. Based on our previous study showing UHMWPE wear debris generation leads to inflammation in the periprosthetic annulus fibrosus [41], we theorized that immune reactions to wear debris will result in the production of key inflammatory/vascular/innervation factors that contribute to the abnormal or enhanced pain sensitization in patients undergoing TDR.

Studies have suggested there is a functional link between the immune response and neurologic changes that ultimately result in the generation of peripheral pain. Specifically, activated macrophages can contribute to experimental pain states by releasing proinflammatory cytokines such as tumor necrosis factor- $\alpha$ (TNF $\alpha)$ and interleukin (IL)-1 $\beta[22,33,37]$; these are both increased in the nucleus pulposus and annulus fibrosus of painful degenerative discs $[17,42]$. It is important to note that $\mathrm{TNF} \alpha$ and IL-1ß are not only potent stimulators of proinflammatory reactions, but both have the potential to induce neural ingrowth into the disc and mediate pain sensitization by upregulating the expression of factors such as nerve growth factor (NGF) and substance P [1]. In addition, TNF $\alpha$ and IL-1ß can directly stimulate pain by acting on nociceptors, sensory neurons that respond by sending signals to the brain that initiate the perception of pain $[36,46]$. Furthermore, these factors can induce angiogenesis by stimulating the release of vascular endothelial growth factor (VEGF), platelet-derived growth factor-bb (PDGFbb), and fibroblast growth factor $[4,38]$. Although the underlying mechanisms of vascular ingrowth remain unclear, VEGF can promote blood vessel expansion into the disc space and subsequently enhance innervation as the growing vessels provide a conduit for ingrowing neurons [27]. Thus, it is feasible that all of these factors may play a role in the peripheral mediation of pain experienced by a subgroup of patients after TDR. 
In this study, we evaluated periprosthetic tissues from patients with lumbar pain collected at the time of TDR revision surgery using immunohistochemistry to quantify the levels of select inflammatory factors known to be involved in both direct and indirect mediation of pain. In addition, we evaluated the association of these factors with the amount of wear debris and the number of macrophages and blood vessels. The purpose of this study was to determine whether (1) periprosthetic UHMWPE wear debris induces immune responses that lead to the production of TNF $\alpha$ and IL-1 1 , the vascularization factors, VEGF and PDGFbb, and the innervation/pain factors, NGF and substance P; (2) the number of macrophages is associated with the production of the aforementioned factors; and (3) the wear debris-induced inflammatory pathogenesis involves an increase in vascularization and associated innervation. Identifying the presence of these factors in lumbar spine tissues could provide valuable insight into the mechanisms that potentially contribute to the development of pain in patients who have undergone TDR.

\section{Materials and Methods}

Lumbar spine tissues from regions adjacent to the implanted device were obtained at the time of revision surgery. Periprosthetic tissues, along with their respective devices, were collected as part of a public and institutional review board-approved, multicenter retrieval research program initiated in $2004[14,15]$. This study is an extension of our previous work that investigated our collection of 11 patients revised for pain after receiving contemporary TDR devices that incorporated cores made of $\gamma$-inert-sterilized UHMWPE GUR 1020 resin; information on the gross appearance of the removed discs and its association with the presence of wear debris was reported in the previous investigation [41]. Visual analog scale (VAS) pain scores are provided, when available (Table 1). The implantation time of the contemporary TDR retrievals for these 11 patients ranged from 1 to 6 years (mean, 3.5 years). Although the original TDR implantation surgery was successful in initially alleviating pain arising from degenerative disc pathology, all were eventually revised primarily for chronic and persistent back pain reemerging at the treated level as early as 6 months. There were no infections. One patient with a single-level TDR and the only patient with a bilevel TDR also showed signs of osteolysis, which is a rare occurrence [40]. Implant migration or subsidence was a complication noted in four of the 11 patients; three of these four were indicated for malpositioning.

In addition to collecting periprosthetic annulus fibrosus tissue samples $(\mathrm{n}=30)$ from 11 patients who received
TDRs, degenerative disc tissue samples $(\mathrm{n}=3)$ from either L4/L5 or L5/S1 segments were obtained from three patients with degenerative disc disease (DDD) who exhibited pain at the time of initial TDR surgery; and intervertebral disc (IVD) tissue samples $(\mathrm{n}=4)$ were obtained from either L4/L5 or L5/S1 at autopsy from four individuals with no clinical history of back surgery or lower back pain (Table 1). Patients' age for both control groups fell within the range of the 11 patients who received TDRs (22-56 years). Normal IVD tissues samples were obtained from the Cooperative Human Tissue Network (CHTN) of the National Cancer Institute (NCI), the National Institutes of Health, Bethesda, MD, USA (https:// chtn.org/). Additional IVD tissue samples were obtained from the Life Legacy Foundation (Tucson, AZ, USA). Visual analog scale pain scores and other patient demographic data were collected for all cohorts when available.

\section{Histological Analysis}

Tissues collected from revision surgeries, primary surgeries for treatment of DDD, and autopsy were fixed in either formalin or Universal Molecular Fixative (UMFIX; Sakura Finetek USA, Inc, Torrance, CA, USA). One to two 4-mm punches from each tissue, considering variations in color, texture, and size of specimen, were embedded in paraffin blocks, and $6-\mu \mathrm{m}$ serial sections were mounted onto ProbeOnPlus (Fisher Scientific Co, Pittsburgh, PA, USA) slides. For the initial analysis studying associations among the inflammation, vasculature, and pain factors in the periprosthetic spine, 30 periprosthetic tissue samples were evaluated from 11 patients who received TDR. For the investigations focused on the localization of blood vessels, inflammatory factors, and macrophages, five representative tissue sections were chosen from five patients undergoing TDR.

The inclusion criteria for this selection process were (1) the presence of wear debris to specifically study wear-induced tissue responses; (2) the presence of at least 10 blood vessels per section to match baseline levels of vascularization noted in intervertebral disc tissue controls; and (3) a range of low to high vascularity based on the Oxford scoring system to study any temporal differences that may be eminent [10].

\section{Wear Particle Analysis}

For UHMWPE particle analysis, mercury-free Harris hematoxylin and eosin (ThermoFisher Scientific, Waltham, MA, USA)-stained tissue sections were evaluated at $\times 200$ magnification for detectable wear debris as small as 0.35 


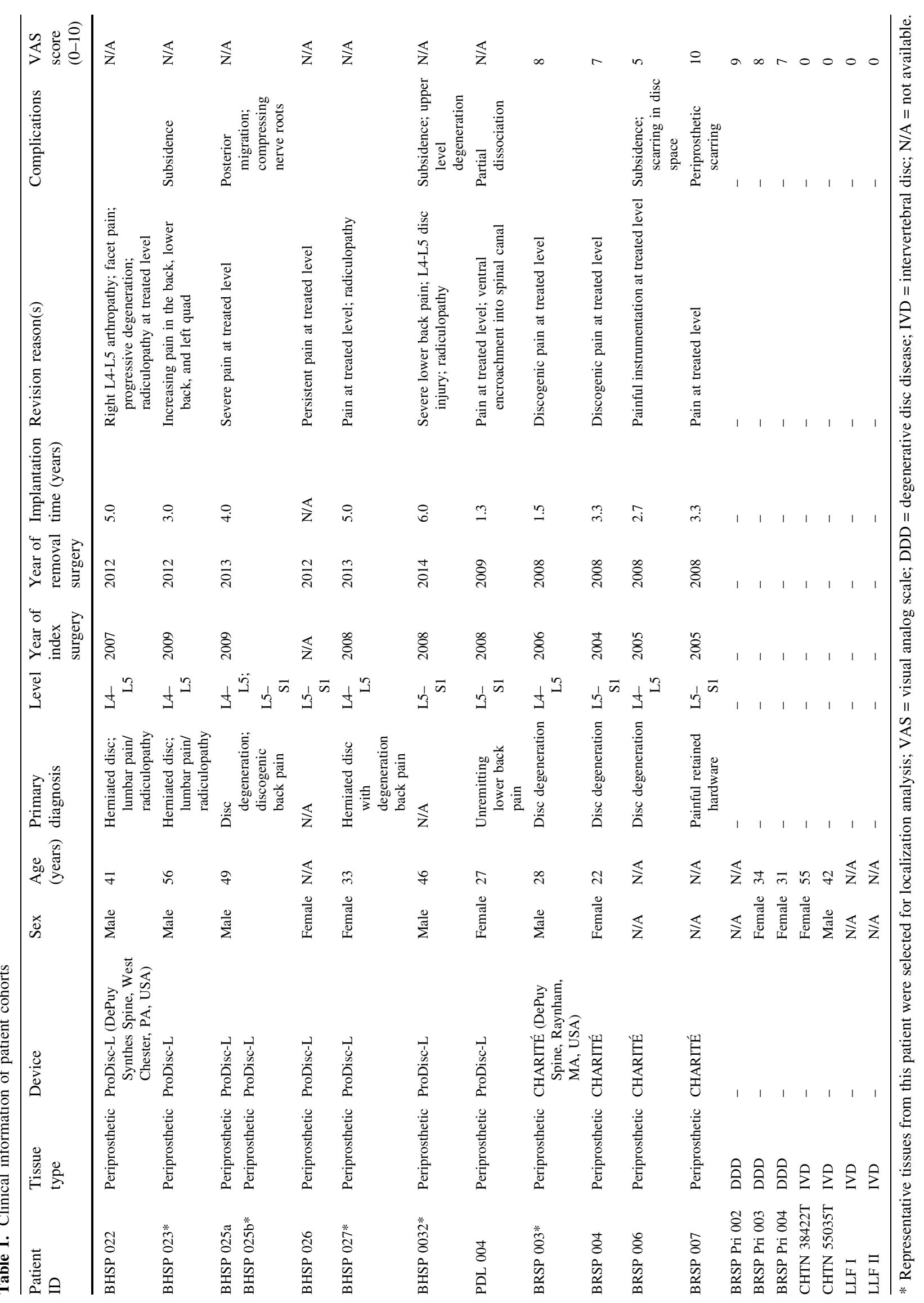


$\mu \mathrm{m}$ (diameter); this size range was determined to be applicable based on previously validated findings on TDR particles ranging from 0.05 to $2 \mu \mathrm{m}$ (mean, $0.46 \pm 0.05$ $\mu \mathrm{m})[29,30]$. In brief, a 36-image (×200 magnification) composite was created from each tissue section under polarized light that corresponded to the transmitted light tissue composites. In each individual image, UHMWPE wear particle number was determined by first using a customized image threshold operation programmed in MATLAB ${ }^{\circledR}$ (MathWorks Inc, Natick, MA, USA) followed by counting/measuring particles using NIH ImageJ (National Institutes of Health, Bethesda, MD, USA).

\section{Immunohistochemistry}

Immunohistochemistry was performed on serial sections to evaluate the expression of six secretory factors and a panmacrophage marker: proinflammatory cytokines, $\mathrm{TNF} \alpha$ (Rabbit IgG, NBP1-19532; Novus Biologicals, Littleton, CO, USA) and IL-1B (Rabbit IgG, AB2105; Abcam, Cambridge, MA, USA); vascularization factors, VEGF (Rabbit IgG, sc-507; SantaCruz, Dallas, TX, USA) and PDGFbb (Rabbit IgG; Abcam); pain-related factors, NGF (Rabbit IgG, AB6199; Abcam), substance P (Rabbit IgG, AB1566; EMD Millipore, Gibbstown, NJ, USA); and macrophage marker, CD68 (Rabbit IgG, AB125157; Abcam). The best antibody working concentration, which gives maximal staining with minimal background, was determined by titration experiments for each of the antibodies. A series of dilutions for each antibody was tested in tissue samples known to be positive for the respective protein: inflammatory and pain-related factors were tested in periprosthetic tissues of patients undergoing total hip arthroplasty (THA) who had severe pain and wear debris; vascularization factors were tested in mouse kidney tissues; and macrophage markers were tested in human tonsil tissues. The optimized antibody working concentrations were: TNF $\alpha$ 1:100, IL-1ß 1:400, VEGF 1:100, PDGFbb 1:100, NGF 1:500, substance P 1:500, and CD68 1:100. Slides with tissues originally fixated in formalin, as opposed to UMFIX, were first treated with an antigen retrieval solution (Vector Labs). All slides were incubated in $0.5 \%$ Triton in phosphate-buffered saline (PBS) to enhance permeability, $3 \% \mathrm{H}_{2} \mathrm{O}_{2}$ in methanol to block endogenous peroxidases, and to block nonspecific background in $4 \%$ bovine serum albumin and $0.1 \%$ Tween 20 in PBS. Lastly, slides were incubated at $4{ }^{\circ} \mathrm{C}$ overnight with the primary antibodies. For antibody visualization, samples were incubated with a pan-specific secondary antibody followed by horseradish peroxidase (Santa Cruz Biotech) and 3,3'-diaminobenzidine (DAB) solution (Vector Labs) and then counterstained with $50 \%$ hematoxylin.
Imaging and Analysis

Each stained tissue section was imaged $(\times 200$ objective $)$ using an Olympus BX50 microscope (Olympus, Melville, NY, USA) equipped with a stepper motor-controlled stage. DAB immunostain was detected by first using a customized image threshold operation programmed in MATLAB $^{\circledR}$ (MathWorks Inc) followed by measuring area through NIH ImageJ (National Institutes of Health). In brief, the red, green, and blue channels for the 24-bit bright field DAB-labeled images were normalized by the sum of the three channels. Pixel values for eight-bit images were calculated using a published formula that allows for maximal separation of DAB-stained pixels from the background tissue: $255^{*}$ blue/ (red + green + blue) [5]. CD68-positive macrophages were quantified in each image $(\times 200)$ of the stained tissue sections with the aid of Image-Pro Plus 6.0 (Media Cybernetics, Silver Spring, MD, USA). A customized macro was generated to count DAB-stained cells. A quantitative value of the inflammatory response was then presented as the number of positive cells (DAB) normalized to total area. In brief, images were split into three eight-bit channels and signal from blue channels was converted into masks based on a threshold value relative to the average signal intensity of each image. Next, count/size operations were used along with watershed split commands to maximize accuracy of counts.

Automation for the quantification of blood vessels was not possible through conventional thresholding and edge detection. Large blood vessels were quantified through manual counts conducted and reconciled by at least two individuals (SYV, MJS). For localization analysis, the tunics of blood vessels were manually traced in each image, creating regions of interest that could be masked. Note that the tunics are not visible at $\times 200$ magnification for smaller vessels such as capillaries, postcapillary venules, or arterioles. However, the focus of the analysis was to determine the number of large vessels (ie, arteries and veins) that theoretically provide a conduit for in-growing nerve fibers [9, 26]. All analysis was performed in a blinded fashion.

\section{Statistical Analysis}

The normality of the data was determined using the Shapiro-Wilk test (IBM SPSS Statistics V22 software package; IBM Corporation, Armonk, NY, USA). To statistically compare immunohistochemical levels between different patient groups, the Mann-Whitney U-test was used. Significance was based on $\mathrm{p}<0.05$. Correlations for wear debris, inflammatory cells, and the six immunohistologic markers were determined using the Spearman Rho correlation test for nonparametric data. Significance was based on $\mathrm{p}<0.05$. 


\section{Results}

\section{Mean Factor Expression in Patient Tissues}

The production of inflammatory, vascularization, and innervation/pain factors was generally higher in TDR patient tissues with detectable wear debris, and the expression of each factor correlated with the amount of wear debris, except PDGFbb. After determining nonnormal distribution of data, nonparametric analysis showed the mean percent area of expression for VEGF $(3.02 \pm 1.01$ versus $0.34 \pm 0.29, \mathrm{p}=$ $0.04)$, IL-1ß (2.41 \pm 0.66 versus $0.13 \pm 0.13, p=0.01)$, and substance $P(4.15 \pm 1.01$ versus $1.05 \pm 0.46, p=0.01)$ was higher in TDR tissues when compared with DDD patient tissues (Fig. 1A-B). Mean percent area expression of all six factors, $\mathrm{TNF} \alpha(5.17 \pm 1.76$ versus $0.05 \pm 0.03, \mathrm{p}=0.02)$, IL$1 \beta(2.41 \pm 0.66$ versus $0.08 \pm 0.002, \mathrm{p}=0.02), \operatorname{VEGF}(3.02$ \pm 1.01 versus $0.02 \pm 0.002, \mathrm{p}=0.02), \mathrm{PDGFbb}(1.80 \pm 0.73$ versus $0.02 \pm 0.02, \mathrm{p}=0.02), \mathrm{NGF}(2.55 \pm 0.87$ versus 0.02 $\pm 0.02, \mathrm{p}=0.02)$, and substance $\mathrm{P}(4.15 \pm 1.01$ versus $0.08 \pm$ $0.04, \mathrm{p}=0.02)$ in TDR tissues was higher compared with normal disc tissues (Fig. 1A, 1C). No differences were observed between DDD and normal disc tissues. Based on wear debris comparisons, the mean factor expression of $\mathrm{TNF} \alpha(6.80 \pm 2.20$ versus $1.91 \pm 0.60, \mathrm{p}=0.02), \mathrm{IL}-1 ß(2.99$ \pm 0.70 versus $1.20 \pm 0.50, \mathrm{p}=0.04), \operatorname{VEGF}(3.65 \pm 1.29$ versus $1.77 \pm 0.84, \mathrm{p}=0.01), \mathrm{NGF}(3.20 \pm 1.12$ versus 1.25 $\pm 0.48, \mathrm{p}=0.04)$, and substance $\mathrm{P}(5.01 \pm 1.22$ versus $2.42 \pm$ $0.90, \mathrm{p}=0.004)$ was higher in TDR tissues with UHMWPE wear particles $(n=14)$ when compared with tissues without particles $(n=16)$ (Fig. 1D). The quantity of wear debris showed a moderately positive correlation with $\mathrm{TNF} \alpha(\mathrm{p}<$ $0.001, \rho=0.63)$ and IL-1ß ( $p=0.015, \rho=0.50$; Fig. 2A). Both factors were expressed at substantially lower levels when no particles were detected with the exception of necrotic tissues (statistically high outliers). Additional wear debris comparisons revealed a moderately positive correlation with VEGF ( $\mathrm{p}=0.003, \rho=0.5)$ but not PDGFbb $(\mathrm{p}=$ 0.17, $\rho=0.3$ ) (Fig. 2B). Lastly, comparisons of NGF and substance $\mathrm{P}$ expression and wear debris showed the amount of wear debris had a positive correlation with both factors $(p=0.012, \rho=0.46 ; p<0.001, \rho=0.59$; Fig. 2C).

Macrophages and Factor Production in Periprosthetic Tissues

Not all tissue sections contained detectable wear particles, but those tissues with wear debris (with the exception of necrotic sections from patient PDL004) were from patients who exhibited implant damage; these tissues showed localized macrophage infiltration (CD68-positive cells), which correlated with the amount of detectable particles
(Fig. 3A-C). The number of macrophages had a strong positive correlation to the amount of TNF $\alpha$ and IL-1ß in these tissues $(p<0.001, \rho=0.85 ; p=0.001, \rho=0.69$; Fig. 4A) and VEGF ( $p=0.001, \rho=0.71)$; however, there was a poor relationship with PDGFbb $(p=0.090, \rho=0.40)$ (Fig. 4B). Lastly, the number of macrophages showed a positive correlation to NGF and substance $\mathrm{P}(\mathrm{p}=0.003, \rho=$ $0.63 ; \mathrm{p}=0.002, \rho=0.65$; Fig. 4C). Fibroblasts (first inflammatory responders) were also qualitatively observed to produce TNF $\alpha$, IL-1ß, VEGF, NGF, and substance P (Supplemental Fig. 1 [Supplemental materials are available with the online version of $\operatorname{CORR}^{\circledR}$.]).

Vascularization and Localization of Factors to Blood Vessel and Nerve Cells in Periprosthetic Tissues

Increased vascularization of periprosthetic tissues was associated with the production of inflammatory, vascular, and innervation/pain factors, and the innervation/pain factors were localized to blood vessels/potential nerve fibers. The number of blood vessels showed a strongly positive correlation to the percent area of $\mathrm{TNF} \alpha(\mathrm{p}=0.001, \rho=$ $0.70)$, a moderate correlation to IL-1B ( $p=0.002, \rho=0.57$; Fig. 5A), and an expectedly strong correlation to VEGF $(\mathrm{p}<0.001, \rho=0.70$; Fig. 5B). There was a weakly positive correlation to PDGFbb ( $p=0.022, \rho=0.46$; Fig. 5B). In the majority of cases, high levels of TNFa and PDGFbb were observed only when the tissue sections were highly vascularized ( $>45$ blood vessels). Furthermore, blood vessel number also showed a strongly positive correlation with NGF ( $p<0.001, \rho=0.70)$ and a moderately positive correlation to substance $\mathrm{P}(\mathrm{p}=0.003, \rho=0.57$; Fig. $5 \mathrm{C})$. Finally, masking blood vessels in images from five representative tissue sections resulted in a decrease in all six factors (Fig. 6A-C). Quantifying the percentage decrease showed that PDGFbb, NGF, and substance $\mathrm{P}$ were reduced by $>25 \%$, inferring they were largely being produced by endothelial cells, vascular smooth muscle cells, and/or peripheral nerves (Fig. 6D). When comparing factor amounts, NGF was higher in blood vessels compared with TNF $\alpha$ ( $p=0.016)$, and PDGFbb was higher in blood vessels compared with VEGF, TNF $\alpha$, IL-1ß, and substance P, respectively $(p=0.008, \rho=0.008 ; p=0.016, \rho=0.016)$.

\section{Discussion}

The pathophysiology of low back pain remains poorly understood, and even less is known about the mechanism(s) involved in the development of pain after metal-onUHMWPE TDR. In this study, we evaluated periprosthetic tissues collected from patients undergoing TDR revised 

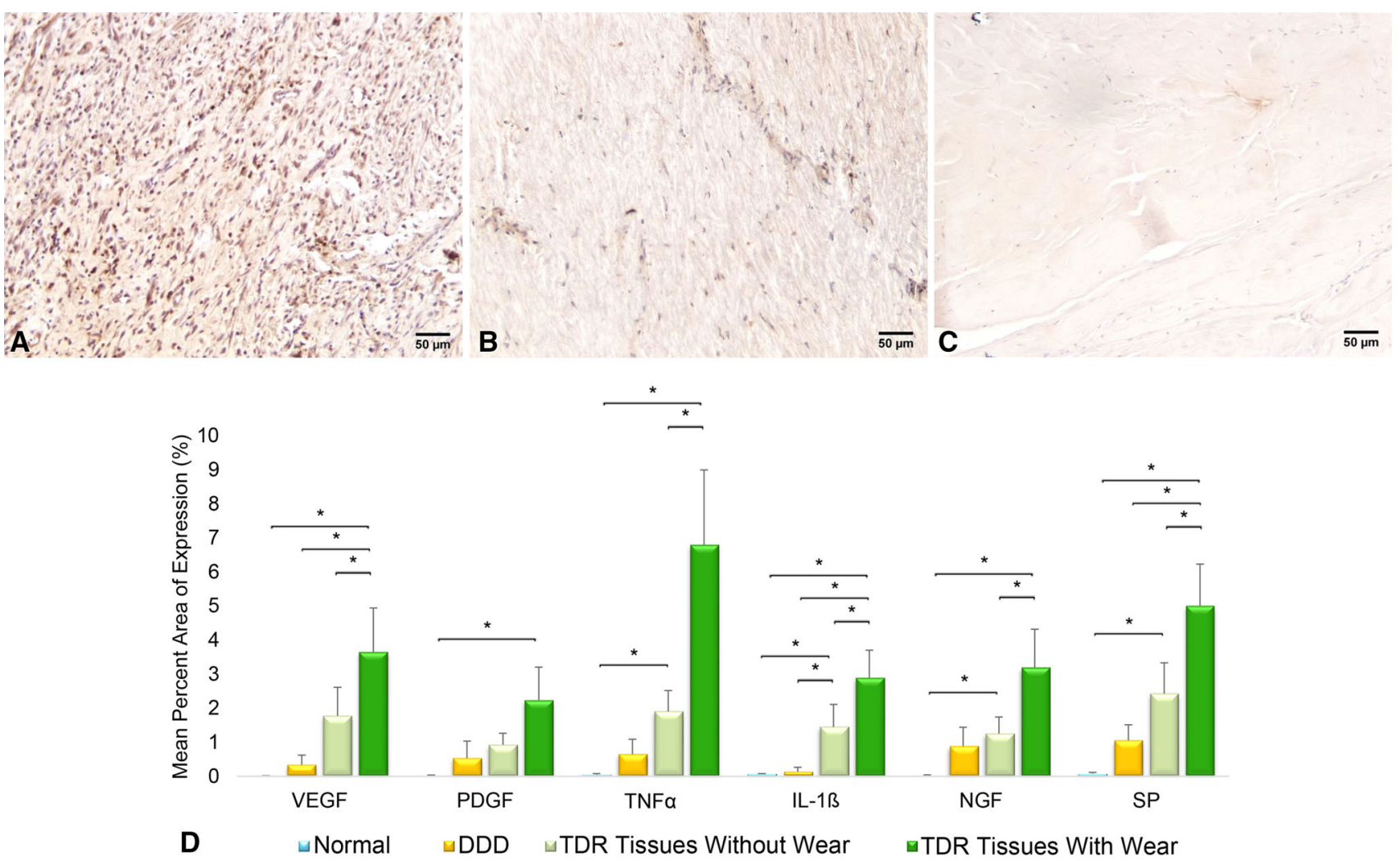

Fig. 1A-D Representative immunostaining is shown for IL-1ß in (A) TDR, (B) DDD, and (C) normal intervertebral disc tissue sections. The graph (D) shows mean expression \pm SD of inflammatory, vascularization, and innervation/pain factors in TDR tissues with $(\mathrm{n}=$ $14)$ and without $(\mathrm{n}=16)$ detectable $(>0.35 \mu \mathrm{m})$ wear debris, DDD $(n=3)$, and normal patient tissues obtained at autopsy $(n=4) .{ }^{*} \mathrm{p}<$ 0.05 . Sections were immunostained using DAB as the chromogenic substrate and counterstained with $50 \%$ hematoxylin; the scale bar is $50 \mu \mathrm{m} . \mathrm{SP}=$ substance $\mathrm{P}$.
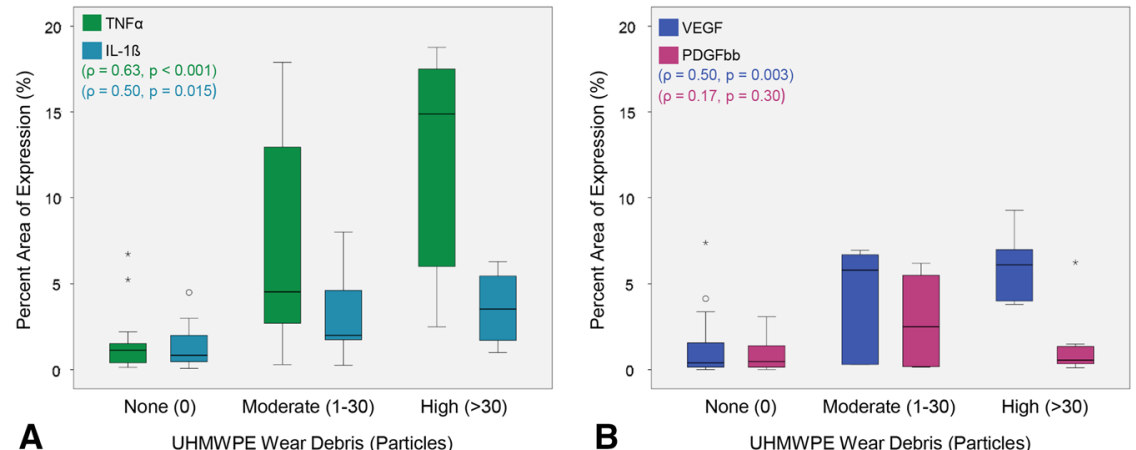

B

UHMWPE Wear Debris (Particles)

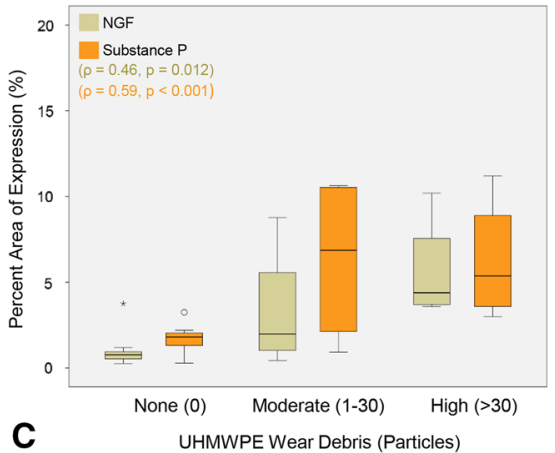

C substance P (SP). There was no correlation with PDGFbb. Data are presented as median $\pm \mathrm{SD}$. The circles and stars represent the outliers and extremes, respectively.

in TDR tissues compared with normal disc tissues from patients with no history of back pain. Three factors, IL-1B, VEGF, and substance $P$, were expressed to a higher extent in TDR tissues compared with tissues from patients with DDD. In addition, TDR tissues with UHMWPE wear debris had increased macrophage infiltration, and both wear debris innervation/pain factors were expressed to a greater extent 

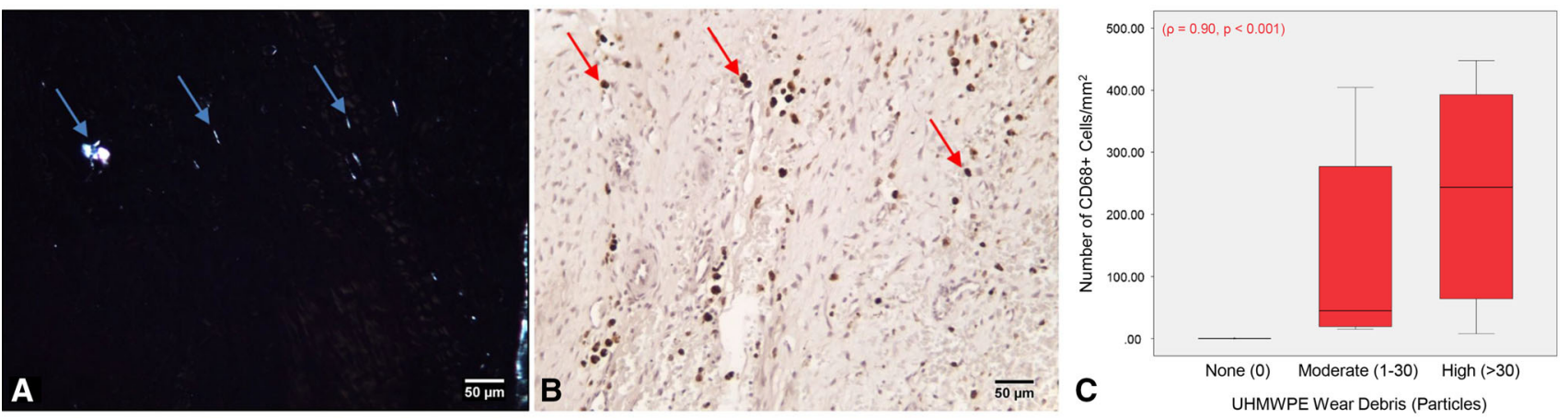

Fig. 3A-C Tissue sections with (A) wear debris contained (B) CD68+ macrophages $(n=30)$. The blue arrows indicate wear particles (polarized light image) and the red arrows macrophages. The graph (C) shows the amount of UHMWPE wear debris strongly correlated with the number of $\mathrm{CD} 68+$ macrophages. Data are presented as median $\pm \mathrm{SD}$. The circles and stars represent the outliers and extremes, respectively. Sections were immunostained using $\mathrm{DAB}$ as the chromogenic substrate and counterstained with $50 \%$ hematoxylin; the scale bar is $50 \mu \mathrm{m}$.
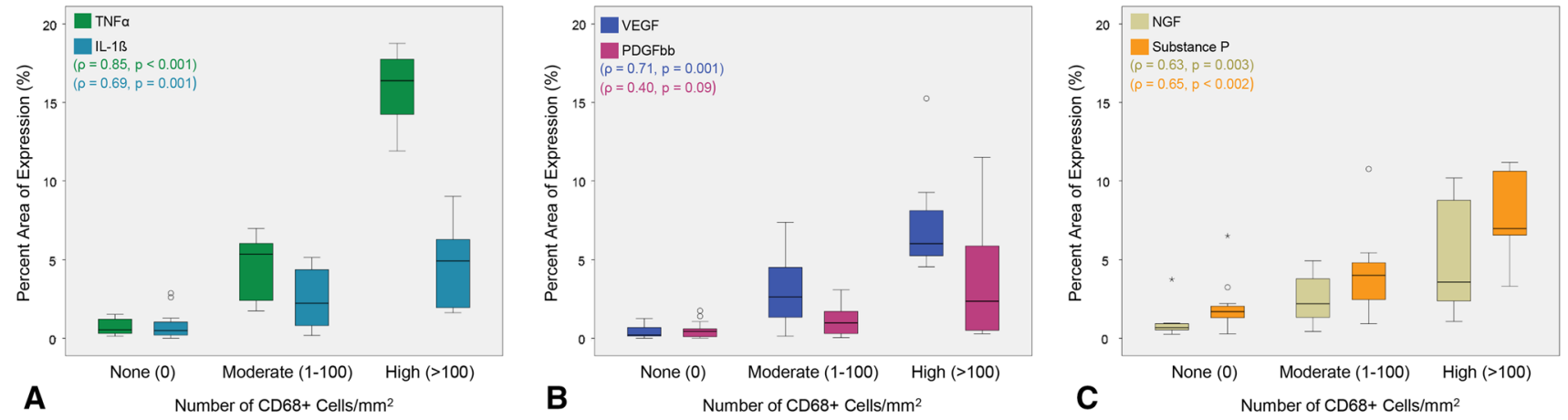

Fig. 4A-C Correlations for the percent area of expression for each inflammatory factor and the number of macrophages in each tissue section $(n=30)$ revealed the CD68+ macrophage number correlated with (A) TNF $\alpha$ and IL-1 $\beta$ and (B) VEGF but not PDGFbb. There was also a correlation for CD68+ cells with (C) NGF and substance P. Data are presented as median $\pm \mathrm{SD}$. The circles and stars represent the outliers and extremes, respectively.
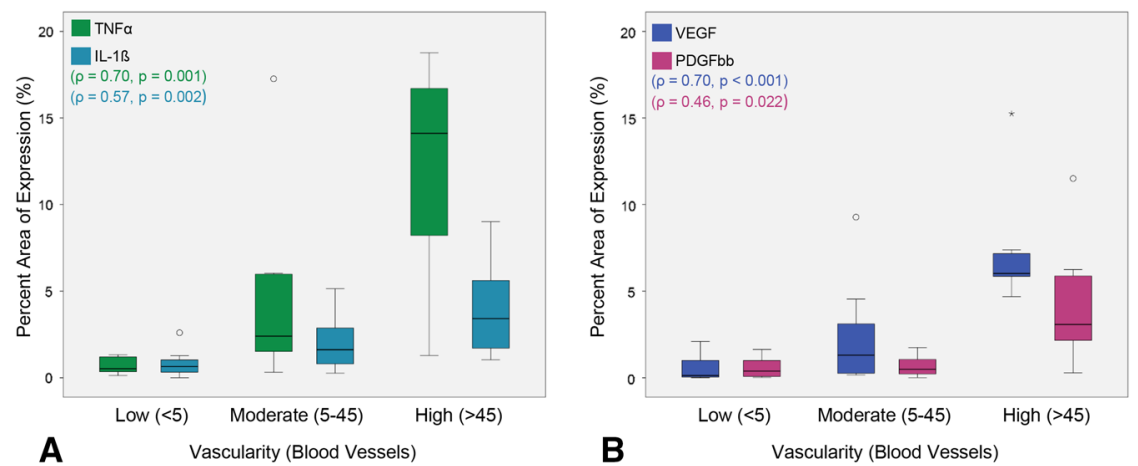

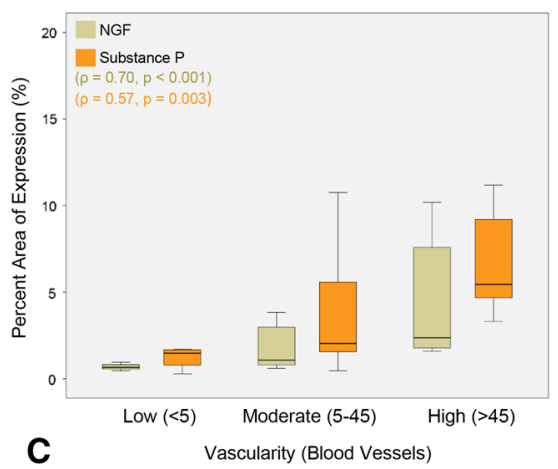

Fig. 5A-C Correlations for the percent area of expression for each inflammatory factor and the number of blood vessels in each tissue section $(n=30)$ revealed the blood vessel number correlated with $(\mathbf{A})$

and macrophages correlated with the amount of TNF $\alpha$, IL$1 \beta$, VEGF, NGF, and substance P, but not PDGFbb. Finally, all of the aforementioned factors correlated with increasing numbers of blood vessels, and NGF and substance $\mathrm{P}$ were localized to vascular/nerve channels. Taken together, this
TNF $\alpha$ and IL-1ß, (B) VEGF and PDGFbb, and (C) NGF and substance $P$. Data are presented as median \pm SD. The circles and stars represent the outliers and extremes, respectively.

study elucidated a part of the pathogenesis of inflammatory particle disease in the periprosthetic spine (Fig. 7), which may provide information needed to identify potential therapeutic targets and treatment strategies to inhibit or mitigate pain sensitization. 

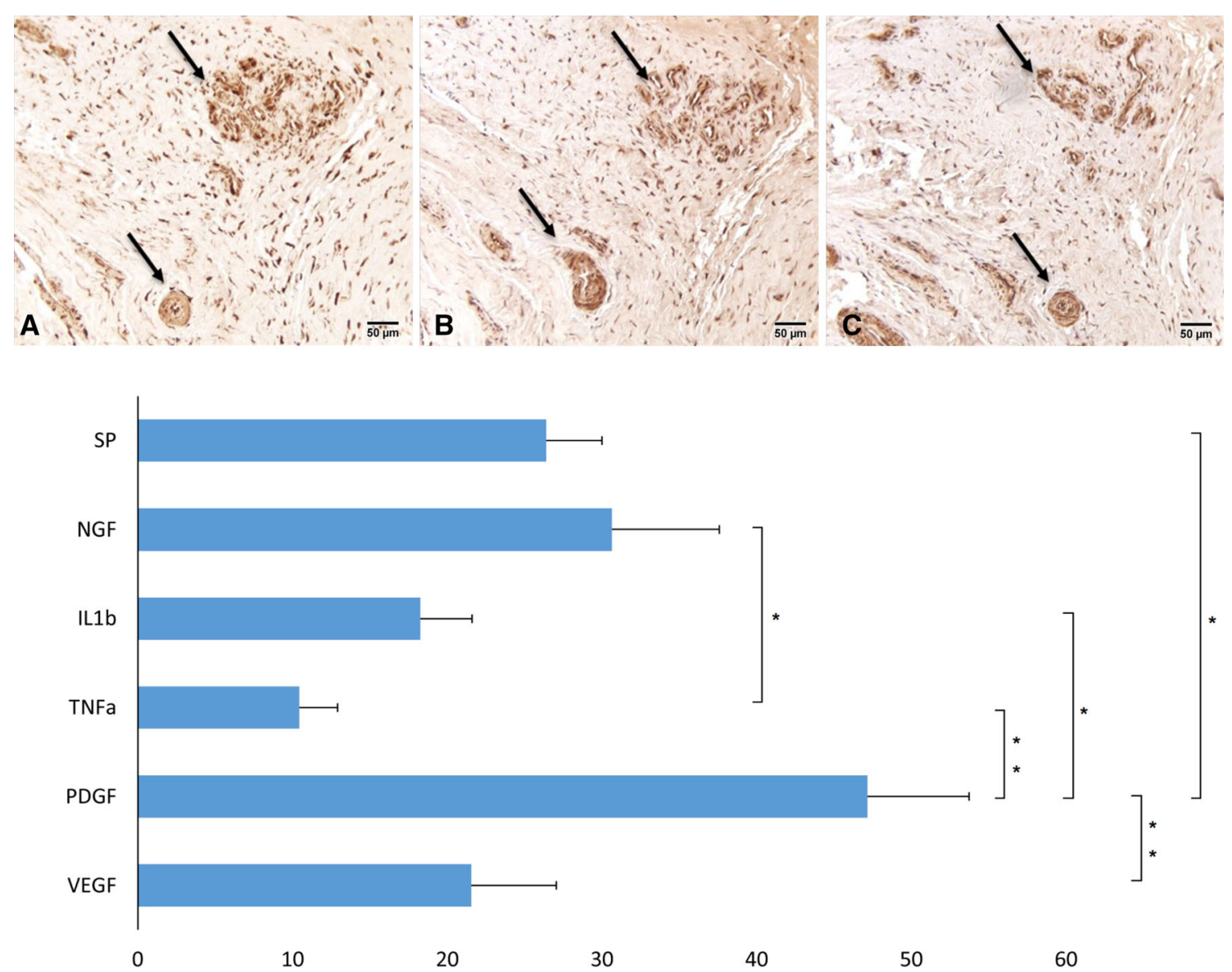

D

Percent Decrease (\%)

Fig. 6A-D Representative images of TDR tissues immunostained for (A) PDGFbb, (B) NGF, and (C) substance P showed colocalization of these factors with blood vessels (black arrows). The graph (D) shows the percentage decrease of all six factors in periprosthetic tissues $(n=$

We acknowledge the limitations of this study. First, although the primary reason for revision for all patients was pain, implant malpositioning or impingement was reported in three patients undergoing TDR; this may serve as a confounding variable because a malpositioned prosthesis can cause foramenal narrowing and compromise the dorsal root ganglion or nerve root, thereby resulting in radiculopathy and pain through physical constriction rather than wear-induced inflammatory pain. However, based on correlation analysis of our limited study sample $(n=3)$, no association was observed between implant complications and the selected factors. VAS pain scores were only available for some patients, making it difficult to establish cause-and-effect relationships with a functional index for pain. Nonetheless, these scores are highly subjective and vary considerably based on individual patient sensitivity to pain. Furthermore, it is worth noting that the surgeon case reports for patients undergoing TDR confirmed that pain was alleviated in all patients after the procedure. It was
5) when blood vessels were masked. PDGFbb, NGF, and substance $P$ were decreased by $>25 \%$. ${ }^{*} \mathrm{p}<0.05$ and ${ }^{* *} \mathrm{p}<0.01$. Sections were immunostained using $\mathrm{DAB}$ as the chromogenic substrate and counterstained with $50 \%$ hematoxylin; the scale bar is $50 \mu \mathrm{m}$.

only after a minimum of 6 months of implantation that pain returned, suggesting another contributing factor (eg, wear debris) was involved.

We were able to detect wear particles as small as 0.35 $\mu \mathrm{m}$; however, a number of in vitro studies has suggested even smaller debris $(0.1-1 \mu \mathrm{m})$ may be present and can stimulate an increased production of inflammatory cytokines [11, 20, 23]. Gamma inert-sterilized UHMWPE components in THA have been shown to generate nanometer-sized wear debris [16, 34]. Because the contemporary TDR is comprised of gamma inert-sterilized UHMWPE, it is possible that smaller wear debris than what was detected in this study are present in these tissues. This may explain the presence of some factors in tissues without detectable wear debris, supporting our hypothesis in all patients undergoing TDR with pain. It is also worth noting that some patients may be more painsensitive to the ingrowth of fewer nerves, and thus not all responses need to be equivalent. Another limitation of our 


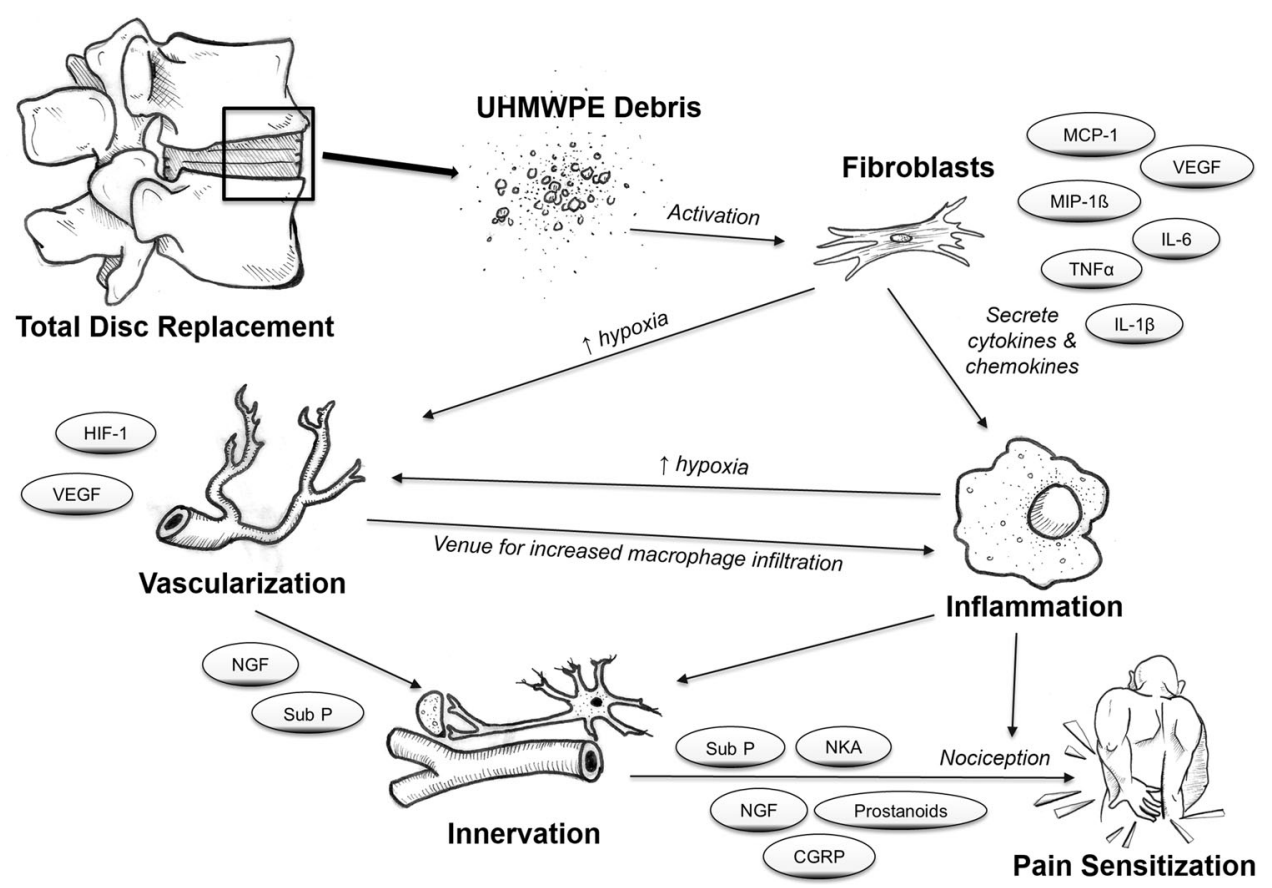

Fig. 7 An illustration of the proposed model of wear debris-induced inflammatory pathogenesis in the lumbar spine. UHMWPE particles activate fibroblasts, which secrete proinflammatory cytokines and chemokines, recruiting inflammatory cells such as macrophages. The increased cellular activity and inflammatory cues induce VEGF secretion and increased vascularization, which can increase

TDR patient cohort is that the implantation times ranged from 1 to 6 years (mean, 3.5 years), thereby representative of only short-term revisions. However, the two patients with implantation times of $\leq 3$ years had a mean of $\geq 20$ particles $/ \mathrm{mm}^{2}$ as described in our previous study [41]. Interestingly, tissue sections with relatively high inflammation and vascularization were often derived from patients with longer implantation times; nonetheless, no correlation was found based on the number of available samples. One other confounding factor is the precise location where the tissues were excised in reference to the UHMWPE core, because the variation in this distance may affect the immune response and thus should be considered in prospective studies. For factor and blood vessel localization analysis, representative tissues from only five of the 11 patients were selected based on our inclusion criteria. Although this may have resulted in overestimation of factor amounts, the criteria allowed us to more accurately identify which factors were localized to blood vessels. Lastly, this study did not identify specific markers for sensory versus nonsensory nerves. Nonetheless, unveiling and linking wear-induced inflammation and innervation/pain factors with increased vascularization provide an important insight into the pathology that may contribute to pain sensitization. inflammation by providing more venues for macrophage infiltration. During vascularization, nerve ingrowth follows the track of newly formed blood vessels, thereby providing an indirect means for nociception. Additionally, inflammation can also exacerbate this process and/or directly result in pain sensitization.

TDR tissues in this study contained higher amounts of the inflammatory, vascularization, and innervation/pain factors than normal disc controls; IL-1ß, VEGF, and substance $\mathrm{P}$ were higher in TDR tissues than DDD controls; and increased expression in TDR tissues was linked to wear debris-induced inflammation. Past studies on total joint replacements of hips and knees suggest that inflammation is in fact initiated by wear debris activation of both fibroblasts and macrophages. Tunyogi-Csapo and colleagues found that fibroblasts in periprosthetic tissues taken from patients with joint replacements secrete proinflammatory cytokines in response to particulate wear as well as the angiogenic factors, VEGF, and fibroblast growth factor [38]. Moreover, the proinflammatory factor recruitment of peripheral blood monocytes and the production of angiogenic factors lead to extensive vascularization of periprosthetic hip tissue [12, 38]. Although we hypothesized a similar upregulation of angiogenic factors, only VEGF was increased and associated with the number of wear particles, whereas increases in PDGFbb were observed only when particle number was high.

Little is known about macrophage and/or fibroblast inflammatory reactions in periprosthetic spine tissues, which may ultimately lead to pain sensitization. Most of what is known about inflammatory reactions to wear debris 
is derived from total joint replacement studies of hips and knees where wear debris (considerably greater amounts compared with TDR) results in osteolysis and is not associated with pain [30]. Therefore, evidence linking wear debris-induced activation of immune cells to pain sensitization would establish a mechanism unique to the physiology of the lumbar spine. It is well accepted from studies on painful DDD that the pathology involves the proliferation of fibroblasts and infiltration of macrophages into the annulus fibrosus in conjunction with the production of pain-associated proteins [4, 8, 43]. Specifically, activated macrophages have been reported to contribute to experimental pain states by releasing factors such as TNF $\alpha$, IL-1 $\beta$, IL-8, NGF, nitric oxide, and prostanoids $[22,33,37]$. In the context of periprosthetic wear debris, joint arthroplasty studies revealed that UHMWPE particles can activate both fibroblasts and macrophages to secrete $\mathrm{TNF} \alpha$ and IL-1ß, which can synergistically contribute to the recruitment of more macrophages [19, 32]. Accordingly, our previous work showed an increased infiltration of macrophages in TDR tissues containing wear particles [41], and this study confirmed that macrophages (and fibroblasts) produced factors that are known to mediate direct or indirect pain.

In this study, increased vascularization was identified as a consistent histomorphologic change in response to wear debris. As such, the ingrowth of blood vessels may be providing a conduit for nociceptive innervation unique to the lumbar spine. Although the current study is the first to our knowledge to identify vascularization and neurologic factors in TDR periprosthetic tissues, studies have reported the presence of proinflammatory factors, blood vessel ingrowth, and nerve ingrowth in patients with DDD with chronic lower back pain $[4,9,24,28]$. Specifically, NGFexpressing blood vessels have been detected and colocalized to sensory nerve fibers in the annulus fibrosus and the nucleus pulposus [9]. These nerve fibers are known to produce neurotransmitters, including substance $P$, involved in pain transmission [3]. Activated macrophages can further exacerbate the condition by triggering the release of neurotrophins and neuropeptides by neighboring macrophages and neurons [1]. Purmessur et al. [31] have shown in vitro that $\mathrm{TNF} \alpha$ stimulation of normal cells from the intervertebral disc increases the production of substance $\mathrm{P}$, whereas IL-1ß stimulation increases NGF. Furthermore, our findings indicate that NGF and substance $\mathrm{P}$ are localized to vascular channels supporting the hypothesis that innervation is intertwined with ingrowing blood vessels. The importance of NGF and substance $\mathrm{P}$ production in periprosthetic tissues is twofold. First, NGF is a known mediator of sensory and nociceptive nerve function and substance $\mathrm{P}$ is a sensory pain-associated neuropeptide released at synapses; thus, both contribute to hyperalgesia (increased sensitivity to pain) [2, 18, 45]. Second, NGF and substance $P$ can contribute to nerve ingrowth [1, 6, 13, 44], and without NGF, all sensory neurons will undergo apoptosis [7]. Both factors work in synergy and may be directly involved in mediating innervation and pain in the lower back [35, 39]. The current findings and previous reports suggest a possible signaling cascade starting with fibroblast activation, macrophage infiltration, increased vascularization, and ultimately innervation/nociception.

In conclusion, by evaluating immunohistochemical markers of inflammation, vascularization, and innervation factors, our findings suggest an UHMWPE wear particleinduced inflammatory response in the spine is linked to pain, the major reason for revision. This is based on the increased presence of inflammatory, vascularization, and innervation factors in TDR patient tissues compared with autopsy samples from patients without pain. Furthermore, a decrease in the number of detectable wear particles resulted in concomitant decreases in factor expression and directly correlated with the number of macrophages and blood vessels. Although this study did not directly measure nerves or pain within the disc regions, the innervating factors NGF and substance $\mathrm{P}$ (which are primarily secreted by neurons) were localized to the vascular channels. Thus, unveiling and linking wear-induced inflammation and innervation factors with increased vascularization provide new clinical insights into the pathology that may contribute to pain sensitization in the spine. Further research with larger cohorts is necessary to fully understand this unique pathophysiology. From a clinical perspective, it is worth noting that TDR hardware from all patients in this study was relatively uncompromised from a mechanical standpoint, and thus, finding ways to either minimize wear debris generation further and/or inhibiting the wear-induced inflammatory pathways will be important in improving TDR clinical outcomes.

Acknowledgments We thank Dr. Kara Spiller for her helpful discussions on macrophage and vascular factors during the course of this study. We also thank medical illustrator, Mike Natter, for his sketch, helping diagram the wear debris-induced inflammatory cascade.

\section{References}

1. Abe Y, Akeda K, An HS, Aoki Y, Pichika R, Muehleman C, Kimura T, Masuda K. Proinflammatory cytokines stimulate the expression of nerve growth factor by human intervertebral disc cells. Spine. 2007;32:635-642.

2. Ahmed M, Bjurholm A, Kreicbergs A, Schultzberg M. Sensory and autonomic innervation of the facet joint in the rat lumbar spine. Spine. 1993;18:2121-2126.

3. Ashton IK, Roberts S, Jaffray DC, Polak JM, Eisenstein SM. Neuropeptides in the human intervertebral disc. J Orthop Res. 1994;12:186-192. 
4. Binch AL, Cole AA, Breakwell LM, Michael AL, Chiverton N, Cross AK, Le Maitre CL. Expression and regulation of neurotrophic and angiogenic factors during human intervertebral disc degeneration. Arthritis Res Ther. 2014;16:416.

5. Brey EM, Lalani Z, Johnston C, Wong M, McIntire LV, Duke PJ, Patrick CW Jr Automated selection of DAB-labeled tissue for immunohistochemical quantification. $J$ Histochem Cytochem. 2003;51:575-584.

6. Edwards RH, Rutter WJ, Hanahan D. Directed expression of NGF to pancreatic beta cells in transgenic mice leads to selective hyperinnervation of the islets. Cell. 1989;58:161-170.

7. Freeman RS, Burch RL, Crowder RJ, Lomb DJ, Schoell MC, Straub JA, Xie L. NGF deprivation-induced gene expression: after ten years, where do we stand? Prog Brain Res. 2004;146:111-126.

8. Freemont AJ, Peacock TE, Goupille P, Hoyland JA, O’Brien J, Jayson MI. Nerve ingrowth into diseased intervertebral disc in chronic back pain. Lancet. 1997;350:178-181.

9. Freemont AJ, Watkins A, Le Maitre C, Baird P, Jeziorska M, Knight MT, Ross ER, O'Brien JP, Hoyland JA. Nerve growth factor expression and innervation of the painful intervertebral disc. J Pathol. 2002;197:286-292.

10. Grammatopoulos G, Pandit H, Kamali A, Maggiani F, GlynJones S, Gill HS, Murray DW, Athanasou N. The correlation of wear with histological features after failed hip resurfacing arthroplasty. J Bone Joint Surg Am. 2013;95:e81.

11. Green TR, Fisher J, Stone M, Wroblewski BM, Ingham E. Polyethylene particles of a 'critical size' are necessary for the induction of cytokines by macrophages in vitro. Biomaterials. 1998;19:2297-2302.

12. Koreny T, Tunyogi-Csapo M, Gal I, Vermes C, Jacobs JJ, Glant TT. The role of fibroblasts and fibroblast-derived factors in periprosthetic osteolysis. Arthritis Rheum. 2006;54:3221-3232.

13. Korsching S, Thoenen H. Nerve growth factor in sympathetic ganglia and corresponding target organs of the rat: correlation with density of sympathetic innervation. Proc Natl Acad Sci U S A. 1983;80:3513-3516.

14. Kurtz S, Steinbeck M, Ianuzzi A, Van Ooij A, Punt I, Isaza J, Ross ER. Retrieval analysis of motion preserving spinal devices and periprosthetic tissues. SAS. 2009;3:161-177.

15. Kurtz SM, van Ooij A, Ross R, de Waal Malefijt J, Peloza J, Ciccarelli L, Villarraga ML. Polyethylene wear and rim fracture in total disc arthroplasty. Spine J. 2007;7:12-21.

16. Lapcikova M, Slouf M, Dybal J, Zolotarevova E, Entlicher G, Pokorny D, Gallo J, Sosna A. Nanometer size wear debris generated from ultra high molecular weight polyethylene in vivo. Wear. 2009;266:349-355.

17. Le Maitre CL, Freemont AJ, Hoyland JA. The role of interleukin1 in the pathogenesis of human intervertebral disc degeneration. Arthritis Res Ther. 2005;7:R732-745.

18. Lewin GR, Ritter AM, Mendell LM. Nerve growth factor-induced hyperalgesia in the neonatal and adult rat. $J$ Neurosci. 1993;13:2136-2148.

19. Lin TH, Kao S, Sato T, Pajarinen J, Zhang R, Loi F, Goodman $\mathrm{SB}$, Yao Z. Exposure of polyethylene particles induces interferon-gamma expression in a natural killer $\mathrm{T}$ lymphocyte and dendritic cell coculture system in vitro: a preliminary study. $J$ Biomed Mater Res A. 2015;103:71-75.

20. Liu A, Richards L, Bladen CL, Ingham E, Fisher J, Tipper JL. The biological response to nanometre-sized polymer particles. Acta Biomater. 2015;23:38-51.

21. Malinsky J. The ontogenetic development of nerve terminations in the intervertebral discs of man. (Histology of intervertebral discs, 11th communication). Acta Anat. 1959;38:96-113.

22. Marchand F, Perretti M, McMahon SB. Role of the immune system in chronic pain. Nat Rev Neurosci. 2005;6:521-532.
23. Matthews JB, Besong AA, Green TR, Stone MH, Wroblewski BM, Fisher J, Ingham E. Evaluation of the response of primary human peripheral blood mononuclear phagocytes to challenge with in vitro generated clinically relevant UHMWPE particles of known size and dose. J Biomed Mater Res. 2000;52:296-307.

24. Melrose J, Roberts S, Smith S, Menage J, Ghosh P. Increased nerve and blood vessel ingrowth associated with proteoglycan depletion in an ovine anular lesion model of experimental disc degeneration. Spine (Phila Pa 1976). 2002;27:1278-1285.

25. Mooney V. Where is the lumbar pain coming from? Ann Med. 1989;21:373-379.

26. Mukouyama YS, Shin D, Britsch S, Taniguchi M, Anderson DJ. Sensory nerves determine the pattern of arterial differentiation and blood vessel branching in the skin. Cell. 2002;109:693-705.

27. Nerlich AG, Schaaf R, Walchli B, Boos N. Temporo-spatial distribution of blood vessels in human lumbar intervertebral discs. Eur Spine J. 2007;16:547-555.

28. Peng B, Wu W, Hou S, Li P, Zhang C, Yang Y. The pathogenesis of discogenic low back pain. J Bone Joint Surg Br. 2005;87:6267.

29. Punt I, Baxter R, van Ooij A, Willems P, van Rhijn L, Kurtz S, Steinbeck M. Submicron sized ultra-high molecular weight polyethylene wear particle analysis from revised SB Charite III total disc replacements. Acta Biomater. 2011;7:3404-3411.

30. Punt IM, Austen S, Cleutjens JP, Kurtz SM, ten Broeke RH, van Rhijn LW, Willems PC, van Ooij A. Are periprosthetic tissue reactions observed after revision of total disc replacement comparable to the reactions observed after total hip or knee revision surgery? Spine (Phila Pa 1976). 2012;37:150-159.

31. Purmessur D, Freemont AJ, Hoyland JA. Expression and regulation of neurotrophins in the nondegenerate and degenerate human intervertebral disc. Arthritis Res Ther. 2008;10:R99.

32. Rao AJ, Gibon E, Ma T, Yao Z, Smith RL, Goodman SB. Revision joint replacement, wear particles, and macrophage polarization. Acta Biomater. 2012;8:2815-2823.

33. Ribeiro RA, Vale ML, Thomazzi SM, Paschoalato AB, Poole $S$, Ferreira SH, Cunha FQ. Involvement of resident macrophages and mast cells in the writhing nociceptive response induced by zymosan and acetic acid in mice. Eur $J$ Pharmacol. 2000;387:111-118.

34. Richards L, Brown C, Stone MH, Fisher J, Ingham E, Tipper JL. Identification of nanometre-sized ultra-high molecular weight polyethylene wear particles in samples retrieved in vivo. $J$ Bone Joint Surg Br. 2008;90:1106-1113.

35. Richardson SM, Doyle P, Minogue BM, Gnanalingham K, Hoyland JA. Increased expression of matrix metalloproteinase10 , nerve growth factor and substance $\mathrm{P}$ in the painful degenerate intervertebral disc. Arthritis Res Ther. 2009;11:R126.

36. Thacker MA, Clark AK, Marchand F, McMahon SB. Pathophysiology of peripheral neuropathic pain: immune cells and molecules. Anesth Analg. 2007;105:838-847.

37. Thomazzi SM, Ribeiro RA, Campos DI, Cunha FQ, Ferreira SH. Tumor necrosis factor, interleukin-1 and interleukin- 8 mediate the nociceptive activity of the supernatant of LPS-stimulated macrophages. Mediators Inflamm. 1997;6:195-200.

38. Tunyogi-Csapo M, Koreny T, Vermes C, Galante JO, Jacobs JJ, Glant TT. Role of fibroblasts and fibroblast-derived growth factors in periprosthetic angiogenesis. J Orthop Res. 2007;25:13781388.

39. Verge VM, Tetzlaff W, Richardson PM, Bisby MA. Correlation between GAP43 and nerve growth factor receptors in rat sensory neurons. J Neurosci. 1990;10:926-934.

40. Veruva SY, Lanman TH, Hanzlik JA, Kurtz SM, Steinbeck MJ. Rare complications of osteolysis and periprosthetic tissue reactions after hybrid and non-hybrid total disc replacement. Eur Spine J. 2015;24(Suppl 4):S494-501. 
41. Veruva SY, Lanman TH, Isaza JE, MacDonald DW, Kurtz SM, Steinbeck MJ. UHMWPE wear debris and tissue reactions are reduced for contemporary designs of lumbar total disc replacements. Clin Orthop Relat Res. 2015;473:987-998.

42. Weiler C, Nerlich AG, Bachmeier BE, Boos N. Expression and distribution of tumor necrosis factor alpha in human lumbar intervertebral discs: a study in surgical specimen and autopsy controls. Spine. 2005;30:44-53.

43. Willems N, Tellegen AR, Bergknut N, Creemers LB, Wolfswinkel J, Freudigmann C, Benz K, Grinwis GC, Tryfonidou
MA, Meij BP. Inflammatory profiles in canine intervertebral disc degeneration. BMC Vet Res. 2016;12:10.

44. Wojtys EM, Beaman DN, Glover RA, Janda D. Innervation of the human knee joint by substance-P fibers. Arthroscopy. 1990;6:254-263.

45. Woolf CJ, Ma QP, Allchorne A, Poole S. Peripheral cell types contributing to the hyperalgesic action of nerve growth factor in inflammation. J Neurosci. 1996;16:2716-2723.

46. Zhang JM, An J. Cytokines, inflammation, and pain. Int Anesthesiol Clin. 2007;45:27-37. 